Plowman, L. \& Stephen, C. (forthcoming). The big picture? Video and the representation of interaction, British Educational Research Journal.

\title{
The big picture? Video and the representation of interaction
}

\author{
LYDIA PLOWMAN \& CHRISTINE STEPHEN \\ INSTITUTE OF EDUCATION \\ UNIVERSITY OF STIRLING
}

lydia.plowman@stir.ac.uk

\begin{abstract}
Researchers who use video to record interactions usually need to translate the video data into another medium at some stage in order to facilitate its analysis and dissemination. This paper considers some methodological issues that arise in this process by examining transcripts, diagrams and pictures as examples of different techniques for representing interaction. These examples are used to identify some general principles for the representation of data where video is the source material. The paper presents an outline of guided interaction and this is used as a case for illustrating these principles in the context of young children, technology and adults in pre-school settings. Although the paper focuses on a specific study and solution, the principles are applicable in all cases where video is used as a source of data for the representation of interaction, whether or not it is technologically mediated.
\end{abstract}

Video is an attractive medium for recording data for researchers who believe that the interactions between people, artefacts and their environment offer insights into learning. Based on the notion that the research setting is directly knowable through what we see, video is considered to provide more potentially illuminating data than questionnaires, interviews or field notes because it appears to represent the complexities of social life and so lend itself to capturing the 'big picture'.

When this data is translated to another medium to facilitate interrogation by researchers or practitioners the very richness of the images produced by video leads to some methodological challenges. The focus here is on two aspects of the transformation of data: during the process of analysis and during the process of representing the outcomes of this analysis for dissemination to others. The paper starts with a description of the research project Interplay:

Play, Learning and ICT in Pre-school Settings because it provides a specific case for exploring these issues and anchors the following discussion. The aim of this research was to identify ways in which practitioners could support children's developing competences with information and communication technologies (ICT) within the context of the playroom. To this end, we recorded interactions between children, technology and practitioners on video and used this data to elaborate our understanding of the concept of 'guided interaction' and to provide illustrative examples for practitioners. We do not focus, however, on the main findings of the research and more detail on both guided interaction and the processes by which we developed this concept in conjunction with practitioners can be found elsewhere (Plowman \& Stephen, 2006; Stephen \& Plowman, under review).

The paper goes on to describe the selection of the most appropriate form of representation for our purposes by examining other methods, including the use of written text, diagrams and pictorial representations. Some principles for representing interaction are derived from this overview and we then return to the specific problem of how to represent guided interaction to illustrate the application of these principles. The representations that we developed to meet the 
requirements of the study use a comic book format and function both as a medium for analysis and for disseminating findings. Although we focus on a specific research project (Interplay) and the conceptual tool derived from that research (guided interaction) the principles are applicable to the representation of other forms of interaction, whether or not they are technologically mediated.

Representation refers to the ways in which images and text reconstruct the original sources (in this case, the interactions and activities of the playroom for the purposes of sharing and interpreting experiences) and, in discussions of visual art, is 'primarily used for the way in which something made may stand for, or bring to mind, some other thing - most typically, for the way in which flat pictures may bring to mind solid bodies' (Bell, 2001). This process of representation is central to how meaning is constructed, both by researchers analysing the data and by those research 'users' to whom the outcomes of analysis are presented. As Erickson (2006) points out, the video itself is not data but a resource for data construction.

Representations are constructed through a process of selecting and excluding data and privileging different modes of communication, thereby presenting different perspectives on 'reality'. Making principled decisions to guide the choice of representation ensures that this process is explicit.

\section{Guided interaction}

Guided interaction is a way of thinking about interactions between children, adults and technology which is central to our analysis of how practitioners can enhance learning with ICT. The term was used in earlier research to refer specifically to computers and the use of interactive media in classrooms and was conceptualized primarily in terms of how design of the interface could support communication between the computer and the student in the absence of teacher support (Plowman, 1992, 1996). However, computer software is not sufficiently intelligent or adaptive to provide adequate support for children and this is particularly apparent when pre-school children, who cannot read instructions and may encounter insuperable operational difficulties, use computers unassisted by adults or others. For Interplay, then, we expanded the concept from its earlier emphasis on design to the role of human help in guiding children's interactions and to a broader range of technologies. Throughout Interplay, guided interaction functioned as a conceptual tool for both practitioners and researchers and its definition evolved over the one-year period of fieldwork and beyond. The main emphasis of this discussion is on the challenges encountered in identifying an appropriate method for the representation of guided interaction but an outline of its characteristics is provided in Appendix 1.

\section{THE RESEARCH SETTING}

Pre-school provision in Scotland, as in many countries, is distinct from formal school education. Practitioners do not view themselves as teachers but see their role as monitoring and responding to children's progress and facilitating development through both the activities they provide and the conversations that prompt and extend children's thinking. Earlier research (Plowman \& Stephen, 2003, 2005) showed that practitioners found the introduction of ICT into the playroom to be challenging: they tended to focus narrowly on computers and there were few examples of direct interactions with children at the computer other than as a reaction to problems such as turn-taking disputes. The culture of pre-school settings values learning through child-initiated pursuits but, without adult support, children did not find their time at the computer rewarding in terms of pleasure or achievement when it was chosen as a free play activity. 


\section{Design of the study}

The first stage of Interplay was to present practitioners with video episodes, recorded in their own nurseries, showing children's interactions with a range of artefacts, some of which were technological and some of which were not. We introduced guided interaction as a way for practitioners to conceptualize ways of supporting children's interactions with ICT and found that, although it was new to them, it was a term with which they felt comfortable. At that time, we used it as a broad term to explore different ways in which practitioners could guide interaction; the detailed characteristics outlined in Appendix 1 are the result of later analysis of video. Practitioners used these group discussions to inform the design of small-scale interventions to explore how they could enhance children's learning with ICT, a process that functioned both as an educational development activity and as a source of data for our research on how learning with ICT could be enhanced. During these interventions, practitioners produced their own records of observations (typically on sticky notes or written in progress files, but occasionally using photographs or video) and we collected more video data on an opportunistic basis. By the end of the fieldwork we had a total of sixteen hours of video recordings made at the eight nurseries which provided illustrations drawn from current practice. The description of guided interaction presented here developed iteratively as a result of our own analysis combined with their experiences and observations. Some of this data was analysed in greater detail towards the end of the study and served the dual purpose of elaborating our understanding of guided interaction and providing exemplar material for dissemination purposes.

\section{Theoretical framework}

Our analytical perspective is informed by sociocultural theory, the key concepts of which can be summarised as agency, action, collaboration and mediation. Within this framework, learning is understood as the result of complex interactions between multiple agents in a cultural context rather than solely as an internal cognitive process. Given our aim of enhancing learning in the playroom we wanted to acknowledge its cultural organisation and the ways in which thinking develops collaboratively through joint mediated activity as well as attend to the ways in which both practitioners and children are active agents in learning. Learning is mediated through cultural tools (Wertsch, 1998) which, in the playroom, may include conversations, social practices and artefacts such as the technologies that we focus on here. Indeed, the concept of guided interaction that we introduced to the practitioners also became a tool that mediated action but, in other contexts, the tool could be a textbook or a whiteboard and learning may be revealed though the ways in which these tools are used. In many environments the main cultural tool is language (Cole \& Engestrom, 1993; Wells, 1999) although, as we shall see, that was not typically the case in this study.

Using mediated action as a focus of analysis is, according to Wertsch (1998, p.17), a way to 'live in the middle' of different theoretical perspectives, being neither solely psychological nor sociological in orientation. This prompts a focus not simply on behaviour or on the individual, but on interaction with the environment, the action being both internal and external and linking cognition and culture. Video enables us to document some of these complex interactions and provides an appropriate tool for mediating our own learning as researchers and practitioners. It does not equip us to identify learning directly and does not provide direct access to cognitive processes; for supplementary data that enables us to make judgements about children's learning we have relied on the practitioners' records. 
Guided interaction is located within a Vygotskian tradition of understanding supported learning and relates to concepts such as scaffolding (Wood, Bruner \& Ross, 1976), assisted performance (Tharp \& Gallimore, 1989), dialogic inquiry (Wells, 1999) and guided participation (Rogoff et al., 1993). Although we observed practitioners scaffolding children's learning in specific circumstances (such as the cookie-making described later), the emphasis on learning through play and exploration, combined with the use of ICT, means that the direct interactions usually associated with these forms of support for learning were unusual (Plowman \& Stephen, 2005). Practitioners' implicit theory of practice derives from a broadly Piagetian view of learning as facilitated by the provision of resources and opportunities (Stephen et al., 2001) and in which play can promote learning by encouraging children to assimilate new materials into existing cognitive structures (Bennett et al., 1997). The role of the adult is interpreted as responding to children's self-initiated activity rather than providing support through an explicit process of co-constructing learning associated with the Vygotskian approaches mentioned above.

All of these approaches can be applied to the support of technology-mediated learning, although they have not been developed specifically for this purpose, but we have identified some distinctive characteristics of guided interaction. Of most relevance here is the role of spoken language as the main mode of scaffolding and other forms of assisted learning. We found that language was not necessarily the main mediator of learning with ICT in a preschool context, particularly when computers were used. In the proximal dimension guided interaction was more often enacted multimodally, through touch, gesture, facial expression or physical presence rather than through talk (see Appendix 1). The concept of task performance which underlies these approaches to assisted learning is inappropriate in the free play context of pre-school settings as guided interaction focuses on the process of learning rather than the successful achievement of a task. And, although these approaches are conceptualized within a sociocultural framework, they tend to focus on the site of engagement, defined as 'the convergence of social practices in a moment in real time' (Scollon, 2001, p.147) and the immediate learning environment rather than the broader spatial and temporal context. Guided interaction refers to practice beyond the proximal adult/child encounters implicit in the approaches indicated above and encompasses planning, the provision of resources and concepts of role. However, these elements are not the subject of focus here as our understanding of this distal dimension was derived from questionnaires and interviews rather than video. The emphasis on learning by doing and the agency of both 'teacher' and 'learner' means that guided interaction has most in common with guided participation, and we draw on Rogoff's ideas about its representation later in the paper. However, the 'interaction' in guided interaction refers specifically to interaction with technology, as well as interaction with the environment and other people. As our earlier studies showed, technology-mediated learning requires specific forms of support.

Nevertheless, all of these concepts of support for learning share an interest in one way or another in the adult's role in mediating learning, particularly:

- the verbal and nonverbal means by which adults guide children

- the verbal and nonverbal means by which children seek guidance

- the use of discourse as a tool for learning

- the role of artefacts in the mediation of learning.

Whether child-to-child, child-to-adult or between people and artefacts interaction is central to understanding learning in a sociocultural framework and all of these approaches describe a 
dynamic, reciprocal process which is multi-causal and highly contingent. Our interest is in looking in more detail at how one realm of interaction can be better understood by finding ways to transform video records of interaction into representations that provide useful information for researchers and for practitioners.

\section{USING VIDEO TO RECORD INTERACTION}

As will be illustrated in the following section, studies that are informed by a sociocultural approach frequently produce transcripts of speech from video because language is seen as one of the main mediators of learning. Language was not as dominant in our analysis because it is not necessarily the main mediational means for children of this age. Talk is a key medium for school classroom interaction (Delamont, 1976) but in pre-school settings responsiveness to children's emotional states is central to the practitioners' role, so touch, gesture and eye contact are also important forms of communication. (See Flewitt, 2006, for a discussion of how these modes of communication are frequently overlooked by researchers in a pre-school context.) We observed few examples of talk in Interplay because children either used the computers on their own or communicated with each other non-verbally by, for example, taking control of the mouse, moving closer when they wanted to join in or upending the sand timer when they wanted to prolong their turn. This lack of talk made video the obvious medium for data collection, although it tends to privilege action and is not well suited to capturing unobservable processes such as thoughts, attitudes, feelings and perceptions which can only be captured obliquely.

Suchman (1987, p.109) claims that video offers a means of considering 'just those fleeting circumstances that our interpretations of action systematically rely upon, but which our accounts of action routinely ignore' and she points out the advantages of using video as a means of providing a record of the action which is independent of the analysis, thus avoiding reliance on field notes. (In Interplay we supplemented the video data with field notes, but this is resource intensive, requiring two researchers in the field. At a later stage, we used practitioners' observations as a way of triangulating data.) Video is relatively straightforward to use when recording people using a photocopier, as in Suchman's study, but playrooms are noisy, busy places where the children are highly mobile. It is difficult to capture the many events going on at the same time and the researcher needs to make constant judgements about the focus of attention and what should be recorded (Jordan \& Henderson, 1995; Plowman, 1999). For instance, practitioners are well practised at regulating children's behaviour by means of glances or gestures from a distance; although pointing the camera at the child will capture their response to this intervention it will not capture the intervention itself so the evidence to which a video account provides access is partial. The danger for researchers is that such decisions about where to locate the focus of attention are made instantaneously, judgements are not made explicit and moments cannot be recaptured if they are missed. Some degree of analysis is therefore taking place even as the material is being recorded but this is not apparent when the material is viewed. The researcher appears to be absent from the data, revealed only when their disembodied voice is heard or by an unsteady picture caused by a hand-held camera. Paradoxically, these intrusions may serve to reinforce the apparent lack of artifice, as they are devices used in fiction and non-fiction films to create the illusion of a direct and unmediated relationship between reality and its representation.

At some stage in the research process this partial semblance of reality is often translated from video into another representation. Finding methods for representing data that are both meaningful for the purpose of interrogation and maintain the appearance of verisimilitude is a challenge that lies at the heart of the ways in which researchers produce and represent 
knowledge, whatever the chosen medium (see Clifford \& Marcus, 1986; Fischman, 2001; Pink, 2001; Rose, 2001; Voithofer, 2005). Researchers often refer to using video to 'capture' complexity (for example Pea, 2006, p. 1323) as if reality can be possessed or seized. Whilst recognizing the impossibility of obtaining complete data, the purpose of the representation is partly to simplify complex processes in such a way that they can be revealed and analysed but information is inevitably lost in this process. Transcription translates the 'raw' data into an easily accessible, paper-based tool for analysis but produces an incomplete representation of the video recording. Analysis then entails extrapolating from the transcript to an even more selective version of activities. Video was nevertheless used as the main form of data capture in our study because this medium:

- provided information that would not necessarily be available from simple observation

- facilitated the analysis and illustration of guided interaction

- enabled practitioners to reflect on practice and on how they could identify opportunities for learning

- provided an opportunity for us to model a technology that practitioners could use for recording their own observations in the playroom.

\section{REPRESENTING INTERACTION}

As Appendix 1 illustrates, guided interaction is multimodal in nature (Scollon \& Levine, 2004), encompassing language, gesture, touch, gaze, and physical movement within a sociocultural framework. These interactions are contingent on the activity, the site of learning and the learner's level of competence and they are influenced by diverse cultural and social processes. Identifying a way to capture multimodal interactions was therefore central to Interplay as we would not be able to identify the characteristics of guided interaction unless we could analyze the range of modalities involved and the ways in which they interact. As part of the process of selecting the most appropriate form of representation for our purposes several different approaches to representing multimodal interactions recorded on video were examined. The first three examples presented here use different versions of conventional written transcripts. This is followed by a description of a Media Interaction Chart and a discussion of two examples of pictorial representations.

\section{Written representations of interaction}

The first example (Cole, 1996, pp.244-245) is from a transcript derived from video of an argument over how many rooms the children have in their apartments and follows on from Dolores saying that Jackie has thirteen rooms. The transcript was produced as part of an attempt to analyse cognitive behaviour across different settings and video was used 'to make visible for analysts the social-interactional constraints on talking and thinking' (op. cit., p. 249). The focus is on speech, with occasional references to gesture or to whom an utterance is directed.

\begin{tabular}{|c|c|c|}
\hline 4 & Dolores & (to Jackie) Count them all (Dolores holds up pinky). \\
\hline 5 & Jackie & Three bathrooms (holds up three fingers). \\
\hline 6 & Dolores & That's not a room. \\
\hline 7 & Reggie & They're rooms. Bath rooms. \\
\hline 8 & Jackie & $\begin{array}{l}\text { No, I'm counting bathrooms. For my (holds out arms) whole } \\
\text { house we got thirteen rooms. There are two (inaudible). }\end{array}$ \\
\hline 9 & Dolores & (holds up 3 fingers) Three, two (adds 2 more fingers). \\
\hline
\end{tabular}


This uses an approach in which spoken language is prioritised but some additional information relating to gesture is provided in brackets. Erickson (2006) refers to the way in which this form of transcription can overprivilege the representation of speech at the cost of nonverbal activity and lead to the concept of social interaction as a ping-pong match. Danby and Baker (2000, pp.115-116) also use conventions that place gesture and body deployment in brackets and integrate this information with the speech. The authors state that the episode demonstrates the pragmatic and strategic moves that children make to build and co-construct their social positions. The following brief example, one of many provided, illustrates the method used for representing the interactions.

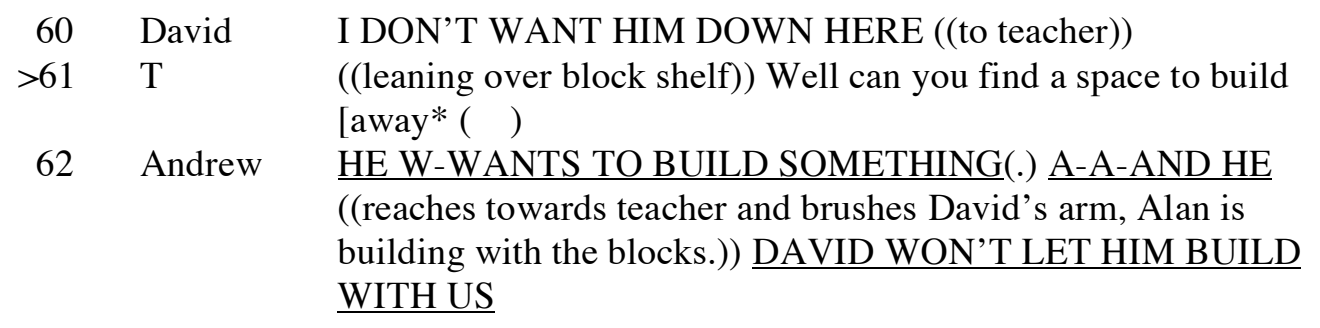

This more detailed transcript is produced in accordance with the principles of conversation analysis, and emphasizes information relating to intonation, gaps and overlaps because it is relevant to the strategies deployed by the boys to maintain or gain social presence. The representation therefore needs to highlight those aspects of interaction that are most relevant to the focus of study.

Bourne and Jewitt (2003, p.67) use video data to analyze the ways in which the interpretation of a literary text is constructed through social interaction in a classroom. They modify standard techniques by adopting a four-way division of information for each utterance. This has the benefit of assigning equal status to verbal and non-verbal interactions, but forming a reconstituted image of the interaction once it has been decomposed like this can be problematic. The teacher is talking in the following excerpt.

Speech: $\quad$ So you see how men then draw a - a kind of - you know

Gaze: $\quad$ at Lizzy

Gesture: right hand in air, pointing brings hands together, palms flat fingers touching [to form a line]

Posture: $\quad$ sitting on edge of desk, one leg on floor, back upright, angled towards Peter

(2) Speech: There's a line between male and female

Gaze: at Peter

Gesture: moves right hand few inches in front of left, palms flat, moves hands away from one another and back again

Posture: $\quad$ sitting on edge of desk, one leg on floor, back upright, angled towards Peter

Each of these three examples is derived from video recordings and uses transcription conventions of varying degrees of detail to represent the range of interactions. It is noticeable that the transcriptions are similar to those used for audio recordings, so even though gestures and gaze are more easily perceived visually, they are represented verbally in these examples ('holds up three fingers'; 'reaches towards teacher'; 'sitting on edge of desk'). Video is presumably selected as a method of data collection for its audio-visual nature but although the 
audio track can be represented in a way that is adequate for most analytical purposes by the verbal format of the transcripts, the visual nature of video is not represented. These transcripts have been decontextualized here, but it is difficult to visualize the context they are designed to represent because all of the information is verbal. It is, of course, possible for the written word to generate images in the mind and the power of both fiction and non-fiction is that it is created anew for each reader, but such texts serve a different explanatory purpose from a transcript, for which interpretation generally needs to be more controlled. For occasions when the author of a text needs to be confident that a reader will recreate a shared image of an event, such as a description of the scene of a crime or accident, it is more usual to combine verbal statements with pictorial records.

Translating video records into text is understandable given that it makes data easier to share amongst researchers and it is a requirement for publication in many academic journals but this is at the cost of losing some of the complexities of interaction. Scollon (2001, p.144) describes how some theories start with a focus on social action but then somehow tend to become focused only on text, claiming that 'representations in text will always under-represent the meanings present in the actions'. A sociocultural perspective in which verbal and non-verbal behaviours have potential communicative significance 'requires more than play-script transcription of speech to make visible the multidimensionality of that ecology' (Erickson, 2004 , p. 203). The next two sections therefore consider ways of supplementing the information provided in transcripts by looking at alternative forms of representation.

\section{Diagrammatic representations of interaction}

Plowman (1992) developed primitive time-based graphical representations of interactions between users and computers in Media Interaction Charts based on video recordings (see Figure 1 for example). These charted guided interaction in its earlier form, showing the interrelationship of design features with group interactions. They provide information from two video sources to allow researchers to get a panoramic view of on-screen events and the situated action of learners both synchronically (at the same time) and diachronically (over a period of time). There is no contextual information apart from that provided in the final column, 'observations and interventions', because the purpose of the charts was to focus quite narrowly on the ways in which design features can support learning.

Figure 1 about here.

These Media Interaction Charts were later developed with Luckin et al. (1998) for other cases of group use of interactive media in schools but they were also labour intensive and suitable only as research tools. The charts could be used to analyze the ways in which design features elicit and foster different kinds of learner response but were not suitable for investigating other factors which could impact on learning. The shortcomings of both approaches include too much emphasis on screen events compared to the students' experiences and wider learning environment and a complex system of coding talk. Where events are broken down into too many component activities like this there is a risk of losing the 'big picture' and they are difficult for non-specialists to interpret. These charts are more visual than the excerpts from the transcripts above and the verbal mode does not dominate in the same way, but they are diagrammatic rather than pictorial. They do not solve the problem of how to facilitate visualization of the context being represented as the information is not sufficiently multimodal. 


\section{Pictorial representations of interaction}

Video is used by Kendon (2004) to produce naturally occurring illustrations of gesture and describes two people reminiscing about their childhoods, one of whom recollects that each year his father, a grocer, would have a very large Christmas cake in the shop which was cut up by weight to sell to customers. As he tells the story he indicates the size of the cake on a table in front of him and then makes a cutting action to demonstrate the shape of the section of cake being cut off. Kendon (p.322) represents this conversation and activity sequence in a series of six simple line drawings based on stills from the video with the dialogue positioned under each diagram (Figure 2). The information in the drawings is limited, as it is the gestures that are the focus of interest, and it is only possible to work out who is speaking and who is listening if the pictures are scrutinized fairly carefully. Key artefacts, such as the table, are sketched in but other contextual information is not included. The diagrams alone are not sufficiently detailed to illustrate how gesture is organized as an activity, how it is organized in relation to speaking and how it contributes to the total meaning of the utterance of which it is a part. A note to the diagrams states 'for an explanation see text'.

Figure 2 about here.

Rogoff (2003, pp.53-61) provides more information in a series of stills taken from a video that has been digitally manipulated to emphasize different foci of attention. In the first image a solitary boy is shown with the background bleached out. In the next image, other people are included but artefacts are not shown and, through the sequence, she demonstrates how the interpersonal, personal and cultural-institutional aspects of the event constitute the activity. She states that it is usually necessary to foreground some aspects of phenomena and background others simply because no one can study everything at once, but these distinctions between foreground and background are made by our analysis rather than being separate entities in reality. The parts of the images that are in focus have a high degree of photorealism, unlike the line drawings used in the example above. Figure 3 shows three people playing Scrabble as a spelling activity organized by the adult. The focus is on the interpersonal interactions, although no convention for indicating speech has been adopted, and detail relating to the classroom context has been faded. The hand holding the lens is a device used by Rogoff to indicate the role of observers and researchers in constructing the focus of analysis.

Figure 3 about here.

The pictorial illustrations produced by Rogoff and Kendon are closer to what we wanted to achieve in representing data from Interplay as they provide visual and contextual information on the use of artefacts. By capturing still images from the video recording they demonstrate that it is possible to analyze the non-verbal interactions that can be so fleeting but which are so important to an analysis of guided interaction in more detail than the standard transcriptions and charts exemplified earlier.

\section{PRINCIPLES FOR REPRESENTING INTERACTION}

This survey of different ways of representing interaction enables us to isolate some overarching principles to be addressed when developing or selecting forms of representation. These principles are relevant regardless of the research context and are presented here before describing the solution formulated for Interplay. The general principles are to i) state an explicit rationale for the format, ii) maintain alignment between the theoretical and empirical approaches and iii) ensure fitness for purpose. 


\section{Rationale for the format}

An unambiguous statement of the rationale for the format chosen to represent interactions is unusual in accounts of research. Whilst it is commonplace to attend to the transcription conventions that are used to indicate pauses and emphasis, it is less usual to see discussions of the format itself compared to alternative forms of representation. More often, a particular format is used by default. Making principled decisions about the chosen representation, whether transcript, chart or picture, reduces the potential for unwittingly privileging a particular theoretical perspective or mode of interaction.

\section{Maintaining alignment between theoretical and empirical approaches}

Although it is now commonplace to take account of social, cultural and institutional settings when theorizing interaction this does not always translate to its representation. In its analysis of situated social practices, the theoretical basis of a sociocultural perspective leads to a focus on activity and mediation rather than the focus on individuals' internalized learning associated with more cognitive approaches. The multimodality of the ways in which this activity is enacted and the ways in which learning is mediated by people and artefacts are not necessarily represented, however, leading to a disjunction between the theoretical orientation and its empirical basis. Heath et al. (2002, p.11) consider that the means by which objects and artefacts feature in the production, coordination and intelligibility of conduct are largely disregarded and comment that 'studies of social interaction remain curiously dislocated from the material circumstances in which it is accomplished'. The representations that we developed are designed to deal with these issues and so are theoretically saturated both in the motivation for their creation and in their reception by others.

This alignment between theory and analysis could be understood as rendering the relationship circular, i.e. that a socioculturally informed approach to transcription might only allow for a sociocultural interpretation of the data, closing down other possibilities. Researchers should be alert to this possibility, although in the case of Interplay the sociocultural orientation has led to more sources of data than might be the case in a more experimental and cognitively oriented study, and so the interpretive possibilities have been expanded rather than constrained. Without the practitioner interviews, discussions at the cluster meetings and their own records, for instance, we would have been unaware of the distal dimensions of guided interaction.

\section{Fitness for purpose}

Representations may range in style from the very complex to the quick and dirty but they need to serve the purpose for which they have been produced. For many researchers, the process of production is very labour intensive because the purpose is primarily analytical and representations are likely to be very detailed. This can also mean that it is difficult for a general audience to interpret the representation (as in the case of the diagrammatic forms described earlier). In our case, they needed to be dual purpose: to assist in the process of identifying and analysing guided interaction and to serve as a source of illustrations for practitioners.

\section{VIDEO AND THE REPRESENTATION OF GUIDED INTERACTION}

In addition to producing these three general principles, the survey of different methods of representing video data enabled us to identify some of the problems inherent in translating from one medium to another. As we had a strong rationale for using video to record data we wanted to maintain as many of its beneficial features as possible. The obvious solution would be to find ways of maintaining the original video format, so avoiding the problem of translation. Olivero, John and Sutherland (2004) propose videopapers as such a solution, web- 
based 'papers' which integrate video sequences with written text and still images into one document. However, a videopaper was inappropriate for our purposes because there is too much information, even in a short video sequence, to enable practitioners to focus on specific behaviours and responses and so identify the characteristics of guided interaction. Suchman's (1987, op . cit.) claim that video enables analysis of 'fleeting circumstances' that we typically do not see depends on watching one frame at a time; such information can easily be overlooked when watching video in real time. On a pragmatic note, many pre-school practitioners either do not have easy access to the web or do not feel confident in using it so videopapers were not appropriate as a form of dissemination.

\section{The analytical process}

The solution that we adopted was to use the video data within a comic strip format. Whilst this may seem flippant in response to the challenges we have identified, our rationale was to find a representational format that served both as an analytical tool and as a means for disseminating findings to practitioners. It also enabled us to align the theoretical and empirical approaches by providing contextual information, demonstrating the role of mediating artefacts, and illustrating the multimodality of interactions. To enable us to do this, we used a hybrid of mediated discourse analysis (Scollon, 2001; Norris, 2004; Norris \& Jones, 2005), mediated action analysis (Wertsch, 1998) and interaction analysis (Jordan \& Henderson, 1995) designed to illuminate our interest in mediated action, context, verbal and non-verbal interaction and support for learning.

The first stage of analysis involved repeated viewing of the video data to select interaction episodes. As the material was going to be used for interpretation by practitioners as well as researchers the selection criteria included pragmatic considerations such as good image quality, an audio track that was clear enough to transcribe accurately and a sufficient number of still frames in a given sequence in which the children were not identifiable. All children gave their verbal consent to the video recordings and all parents gave signed, informed consent for their children to be recorded. However, the permission given was for photographs and video recordings to be used for 'educational purposes'. This was adequate for sharing data with practitioners in different nurseries but is more ambiguous for use in journal papers or web sites and we therefore decided to use only images in which children could not be identified. Other criteria included finding illustrations of a diverse range of ICT in use and examples of interactions that could be classified as productive or unproductive.

The second stage was to code child and practitioner actions in both the selected interaction episodes and sequences that provided interesting data but which were not suitable for dissemination due to the exacting selection criteria. The analysis enabled us to devise a taxonomy of guided interaction showing examples of different types of support, the different modes in which that support is enacted, and the learning that follows from the interactions (Appendix 1). The representations of guided interaction needed to illustrate the complex, dynamic and reciprocal relationship between practitioner and child/ren. To this end, representing guided interaction has been an important part of the process of defining guided interaction as, although we had an initial working definition, this was elaborated through working with the practitioners and identifying the defining features.

Making principled decisions about the choice of format enabled the link between theory and its empirical foundation to be made more explicit and prompted us to articulate the requirements that the representations needed to fulfil. These were to: 
- indicate the ecology of the playroom

- be appropriate for multiple participants

- demonstrate the role of artefacts in mediating interactions

- acknowledge the multimodal nature of social interactions and give equal priority to verbal and non-verbal means of communication

- integrate the technological and interpersonal dimensions of using ICT

- encompass social, cognitive, affective and sensorimotor responses

- balance decomposition of activities with a holistic account of events

- be pictorial rather than based on charts or transcripts

- capture dynamic processes within still images.

Having identified that the comic book format would meet these requirements the final stage was to translate the chosen sequences into the format. It was during this process that we became aware that video recordings had not captured some indirect aspects of guided interaction that we had identified from interviews with practitioners. These were subsequently referred to as being in the distal dimension because they were not visible in the situated interactions, although the activities that were more remote in terms of time and space were also guiding interaction, albeit indirectly. The modes by which support was enacted in the distal dimension were broadly categorised as policy and pedagogy. Policy refers to actions taken in order to comply with policy directives and curriculum guidelines, including planning and recording children's learning, and pedagogy is an umbrella term which refers to the ways in which practice and concepts of role shape actions in the playroom. A fuller description and a table setting out the characteristics of guided interaction in the distal dimension is available in Plowman and Stephen (forthcoming).

In terms of fitness for purpose the representations needed to provide information that was easy to understand and to function both as research tools and as explanatory tools for practitioners. The purpose of the representations was to facilitate a process by which practitioners could identify and interpret what guided interaction is, recognise opportunities to enhance children's encounters with ICT and apply this knowledge in ways that seem unforced and do not disrupt playroom routines. The concept of guided interaction is likely to be more memorable in the comic strip format than if presented as text and, being suitable for distribution on paper as well as on the web, is accessible. Nevertheless, this was a labour intensive process and was just one of several approaches to analysing the data.

\section{Comic book representations of interaction}

The comic strips shown in Figures 4 and 5 are based on some of the video images selected in the process outlined above. (The comic strips were produced with Comic Life software, available from http://plasq.com. Stills from the digital video were imported into iPhoto and then into Comic Life, where different templates and speech bubbles can be selected to produce a multi-panelled comic strip.) Figure 4 was selected to demonstrate how practitioners guide children's interactions in activities that do not include ICT. In this case, the practitioner is showing the children how to make cookies and the artefacts involved include a range of equipment and ingredients for baking: bowls, cookie cutters, baking trays, a rolling pin, flour, butter and a recipe book. The dialogue is represented in the speech bubbles, using different shapes for the practitioner and the children. The commentary in the caption boxes indicates some of the ways in which the children's interactions are being guided and is the outcome of discussions with practitioners combined with the development of the taxonomy of guided interaction (see Appendix 1). This activity was recorded early in the study and the comic strip is used to demonstrate that guiding children's interactions, using explicitly instructional 
language, engaging children in learning conversations and modelling behaviour are all actions that can be appropriate and, in some circumstances, come naturally to practitioners.

Figure 4 about here.

Prior to the small-scale projects introduced by practitioners to explore what forms guided interaction might take within their practice we found such behaviours were unusual with reference to ICT. In these contexts, practitioners were much more reluctant to use an instructional approach, partly because their confidence with some forms of technology was limited. This was particularly noticeable when they were using desktop computers, one reason why we encouraged practitioners to embrace a wider range of both existing and new technologies in the playroom. Figure 5 shows an adult and child using an audio cassette recorder, set up in what is known as the listening centre in most playrooms. This was one of the interventions that had been chosen to explore ways in which children could enhance learning and pleasure from using an existing playroom technology which was often disregarded in favour of computers. The artefacts that have been provided to support the encounter include finger puppets for acting out the story of the three little pigs on the audio cassette, the book in which the story can be followed, dual headphones so that the practitioner can check what the child can hear, and coloured stickers on the machine to indicate the buttons for stopping and starting the tape.

Figure 5 about here.

In both cases, a sequence of six images provides a short narrative that exemplifies different aspects of guided interaction and demonstrates its multimodal nature, showing gesture, speech and presence as means of support. The comic strip format is ideal for representing a dynamic process within a sequence of static images, making it easier to isolate key gestures and responses, non-verbal means of interaction and the ways in which multiple participants orient to each other and to features in the environment. As an analytical tool they make explicit interactions that had not been visible to us in the moment or to practitioners. The frame-byframe process of producing comic strips forces the researcher to identify the key actions and transitions. The sequence of images also enables the researcher and the reader to balance the decomposition of activities necessary for understanding its components with a coherent, narrative account of events. The explicitly constructed nature of this comic strip format emphasizes the researcher's role in producing and presenting knowledge, so partly performing the same function as Rogoff's lens in Figure 3. The use of video can give the illusion of an unmediated reality but the construction of the comic strip highlights the artifice involved in producing a representation of events.

For our purposes, the comic strip format provides a number of advantages over alternative forms of representation, but there are shortcomings. The main problem is that the distal dimension of guided interaction is not fully represented. Almost by definition, the ways in which encounters with technology are supported in the distal dimension (such as planning and recording) are less visible and so less amenable to representation in such a format, although the comic strips produced here show evidence of the provision of resources. This is not surprising given that guided interaction is most visible at the 'site of engagement' (Scollon, 2001, op. cit.) but we also need to conceptualize the site of engagement as extending over time and space. Erickson (2004, p.180) describes how the interactional 'work' is to a large extent determined by the institutional setting but it is difficult to represent this adequately within a format that is easy to interpret, let alone more distant social and cultural influences. 
In common with other sociocultural approaches to studying interaction and learning, it is difficult to know where or how to draw the boundaries of what is relevant for representation and analysis at the macro and the micro levels and it is not surprising that most studies focus on a small number of people and their local environment. If the format does capture elements of the broader context it is likely to be insufficiently detailed for analysis or description at the micro level. Conversely, if the analysis is fine-grained it is likely to lack information about the broader context. Typically, these omissions are made good in the accompanying text rather than in the visual representations. For these reasons, researchers need to be explicit about the principles that underlie the representations they use so that they can ensure that the requirements that they have identified as most important are met and they acknowledge any shortcomings. The comic book format is also a cultural artefact and researchers should be confident that readers will be familiar with its conventions and able to interpret it.

\section{CONCLUSION}

Eisner (1997) discusses text and number as the default representations of what we have learned about the educational world and advocates stories and pictures as two alternatives. Both of these are blended in the comic strips and, whilst relatively new to educational research, stories and pictures have the benefit of being long-established and easily accessible modes of communication. (Other forms he mentions include diagrams, maps, theatre, demonstrations, photography and poetry.) This suggestion that the range of approaches to recording and disseminating data should be broadened has implications for the epistemological grounds of representation for, as Eisner points out, there are good reasons why text is so central to our traditional view of knowledge, given that it is closely associated with verification, truth, claims and assertions. Alternative forms of data representation can illuminate the variety of ways through which our experience is understood and may promise greater accessibility and authenticity as well as 'productive ambiguity' (op. cit., p. 8), in which the material presented is more evocative than denotative. This can generate insight, highlight complexity and increase the probability that multiple perspectives will emerge.

This fluidity of interpretation is what we aimed for in producing representations that would prompt practitioners to reflect on ways in which they could create opportunities for learning with ICT in the playroom. For example, one suggestion for professional development was to leave the caption boxes blank so that practitioners could jointly decide what text they would insert to support learning and this is congruent with our desire to avoid a deficit model of practice. However, such ambiguity has the potential to be one of Eisner's perils of alternative forms of data representation if it means that interpretation is idiosyncratic and imprecise. In our case the video data was viewed and informally analyzed by practitioners so we can have some confidence in our interpretations. The comic strips are not proffered as a solution for others confronting the challenge of representing interactions recorded on video but as an example of how the process of thinking through the principles led to this particular choice of medium. In this case, the process of producing the comic strips was a form of analysis but they also provide a resource for dissemination of the research findings.

Developments in audio-visual and computing technologies facilitate new approaches to recording interactions which are seductive to researchers. The use of wireless microphones and compact digital video cameras have transformed the means of recording data whilst desktop editing software and the use of the web have transformed the means of presentation. The miniaturization of video technology brings increased ease and mobility to data collection but does not necessarily provide access to the 'big picture' alluded to in the title of this paper. The 
focus on activity and mediation which underpins a sociocultural framework requires analysis of people, events and interactions but the use of video necessarily means a focus on the visible and may mean that other, more distal, forms of interaction are overlooked. Engaging with the principles suggested here may enable researchers to prioritize requirements, acknowledging that there are different forms of representation beyond the default written transcript and that these different forms alter our access to the big picture and the ways in which we see and interpret interaction.

\section{Acknowledgements}

The research reported here was conducted as part of Interplay: Play, Learning and ICT in Preschool Education and was funded by the ESRC's Teaching and Learning Research Programme (award RES-139-25-0006). We are grateful to the researchers, Susan Downey and Daniela Sime, and to the children, families and pre-school practitioners who participated in the study. Further information and papers related to the Interplay study are available from www.ioe.stir.ac.uk/interplay. The ESRC's e-Society Programme has funded a development of the work reported here which focuses on pre-school children in their home environment. The study, Entering the e-Society: Young Children's Development of e-Literacies (award RES-34125-0034), is due for completion in 2007. Further information is available from www.ioe.stir.ac.uk/Research/e-Society.

\section{References}

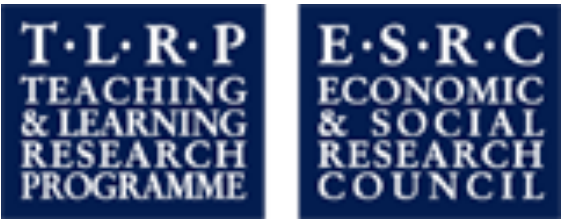

Bell, J. (2001). "representation" in H. Brigstocke (Ed) The Oxford Companion to Western Art (Oxford, Oxford University Press) accessed through Oxford Reference Online 20 May 2006.

Bennett N. Wood L. \& Rogers S. (1997) Teaching Through Play: Teachers' thinking and classroom practice (Buckingham, Open University Press).

Bourne, J. \& Jewitt, C. (2003) Orchestrating debate: a multimodal analysis of classroom interaction, Reading - Literacy and Language, 37 (2) 64-72.

Clifford, J. \& Marcus, G. (1986) Writing Culture: The poetics and politics of ethnography (Berkeley, University of California Press).

Cole, M. (1996) Cultural Psychology: A once and future discipline (Cambridge MA, Harvard University Press).

Cole, M. \& Engestrom, Y. (1993) A cultural historic approach to distributed cognition, in: G. Salomon (Ed) Distributed Cognitions (Cambridge, Cambridge University Press) 1-46.

Danby, S. \& Baker, C. (2000) Unravelling the social order in block area, in: S. Hester \& D. Francis (Eds) Local Educational Order: Ethnomethodological studies of knowledge in action (Amsterdam, John Benjamins) 91-140.

Delamont, S. (1976) Interaction in the Classroom (London, Methuen).

Eisner, E. (1997) The promise and perils of alternative forms of data representation, Educational Researcher, 26 (4) 4-11.

Erickson, F. (2006). Definition and analysis of data from videotape: some research procedures and their rationales, in: J. Green, G. Camilli \& P. Elmore (Eds) Handbook of 
Complementary Methods in Education Research, $3^{\text {rd }}$ edition (Mahwah, NJ, Lawrence Erlbaum).

Erickson, F. (2004) Talk and Social Theory (Cambridge, Polity Press).

Fischman, G. (2001) Reflections about images, visual culture, and educational research, Educational Researcher, 30 (8) 28-33.

Flewitt, R. (2005). Is every child's voice heard? Researching the different ways 3-year-old children communicate and make meaning at home and in a pre-school playgroup, Early Years, 25 (3) 207-222.

Heath, C., Luff, P. vom Lehn, D., Hindmarsh, J. \& Cleverley, J. (2002) Crafting participation: designing ecologies, configuring experience, Visual Communication, 1 (1) 9-33.

Jordan, B. \& Henderson, A. (1995) Interaction Analysis: Foundations and practice, Journal of the Learning Sciences, 4 (1) 39-103.

Kendon, A. (2004) Gesture: Visible action as utterance (Cambridge, Cambridge University Press).

Luckin, R., Plowman, L., Gjedde, L., Laurillard, D., Stratfold, M., \& Taylor, J. (1998) An Evaluator's Toolkit for Tracking Interactivity and Learning, in: M. Oliver (Ed) Innovation in the Evaluation of Learning Technology (London, University of North London) 42-64.

Norris, S. (2004) Analyzing Multimodal Interaction: A methodological framework (London, Routledge).

Norris, S. \& Jones, R. (Eds) (2005) Discourse in Action: Introducing mediated discourse analysis (Abingdon, Routledge).

Olivero, F., John, P. \& Sutherland, R. (2004) Seeing is believing: using videopapers to transform teachers' professional knowledge and practice, Cambridge Journal of Education, 34 (2) 169-176.

Pea, R. (2006). Video-as-data and digital video manipulation techniques for transforming learning sciences research, education, and other cultural practices, in: J. Weiss, J. Nolan, J. Hunsinger, P. Trifonas (Eds) The International Handbook of Virtual Learning Environments (Netherlands, Springer) 1321-1393.

Pink, S. (2001) More visualising, more methodologies: on video, reflexivity and qualitative research, The Sociological Review, 49 (4) 586-599.

Plowman, L. (1999) Using Video for Observing Interaction in the Classroom. Spotlight 72 (Edinburgh, Scottish Council for Research in Education).

Plowman, L. (1996) Narrative, linearity and interactivity: making sense of interactive multimedia, British Journal of Educational Technology, 27 (2) 92-105.

Plowman, L. (1992) An ethnographic approach to analysing navigation and task structure in interactive multimedia: some design issues for group use, in: A. Monk, D. Diaper, MD Harrison (Eds) People and Computers VII (Cambridge, Cambridge University Press) 271-287.

Plowman, L. \& Stephen, C. (forthcoming) Guided interaction in pre-school settings. Submitted to Journal of Computer Assisted Learning. 
Plowman, L. \& Stephen, C. (2006) Technologies and learning in pre-school education. Paper presented at the American Educational Research Association conference, San Francisco, April 2006.

Plowman, L. \& Stephen, C. (2005) Children, play and computers in pre-school education, British Journal of Educational Technology, 36 (2) 145-158.

Plowman, L. \& Stephen, C. (2003) A 'benign addition'? Research on ICT and pre-school children, Journal of Computer Assisted Learning, 19 (2) 149-164.

Rogoff, B. (2003) The Cultural Nature of Human Development (New York, Oxford University Press).

Rogoff, B., Mistry, J., Goncu, A. \& Mosier, C. (1993) Guided Participation in Cultural Activity by Toddlers and Caregivers, Monographs of the Society for Research in Child Development, 58 (8) Serial No. 236.

Rose, G. (2001) Visual Methodologies (London, Sage).

Scollon, R. (2001) Action and text; towards an integrated understanding of the place of text in social (inter)action, mediated discourse analysis and the problem of social action, in: R. Wodak \& M. Meyer (Eds) Methods of Critical Discourse Analysis, (London, Sage) 139183.

Scollon, R. \& Levine, P. (2004) Multimodal discourse analysis as the confluence of discourse and technology, in: P. Levine \& R. Scollon (Eds) Discourse and Technology: Multimodal discourse analysis, (Washington DC, Georgetown University Press) 1-6.

Stephen, C., Brown, S. \& Cope, P. (2001) Alternative perspectives on playroom practice, International Journal of Early Education, 9 (3) 193-205.

Stephen, C. \& Plowman, L. (under review). Enhancing learning with ICT in pre-school playrooms. Submitted to Early Child Development and Care.

Suchman, L. (1987) Plans and Situated Actions: The problem of human-machine communication (Cambridge, Cambridge University Press).

Tharp, R. \& Gallimore, R. (1998) Rousing minds to life: teaching, learning and schooling in social context (Cambridge, Cambridge University Press).

Voithofer, R. (2005) Designing new media education research: the materiality of data, representation and dissemination, Educational Researcher, 34 (9) 3-14.

Wells, G. (1999) Dialogic Inquiry: Toward a sociocultural practice and theory of education (Cambridge, Cambridge University Press).

Wertsch, J. (1998) Mind as Action (New York, Oxford University Press).

Wood, D. J., Bruner, J. S., \& Ross, G. (1976) The role of tutoring in problem solving, Journal of Child Psychology and Psychiatry, 17 (2), 89-100. 


\section{Appendix 1 Characteristics of guided interaction}

There are two main dimensions of the framework for understanding guided interaction: distal and proximal. Distal refers to guided interaction that takes place at a distance from the specific learning interaction and so has an indirect influence on learning. Proximal refers to the face-toface interactions between adults and children that have a direct influence on learning.

The three main areas of learning with technologies in pre-school that can be supported by guided interaction were identified as developing a disposition to learn, extending knowledge of the world and acquiring operational skills. The table produced here refers to proximal guidance because it is aspects of this that the comic strips represent; another table itemises the characteristics of guided interaction in the distal mode but there is no equivalent of the comic strips. All examples in the table are taken from data collected during Interplay: some are drawn from children's use of computers, some are drawn from children's use of other forms of ICT. Full descriptions of the categories of learning, the table reproduced here and another table which categorizes guided interaction in the distal dimension are provided in Plowman \& Stephen (forthcoming).

Table 1: Characteristics of guided interaction

\begin{tabular}{|c|c|c|c|}
\hline $\begin{array}{l}\text { Form of guided } \\
\text { interaction }\end{array}$ & Example & Mode & Learning \\
\hline demonstrating & $\begin{array}{l}\text { how to use a tool such as the paintbrush or eraser } \\
\text { placing a hand over child's hand as they move the } \\
\text { cursor or click on icon } \\
\text { how to frame a picture in viewfinder } \\
\text { how to plug in electronic keyboard } \\
\text { turning over pages of story as children listen on } \\
\text { audio tape } \\
\text { waving hand in front of EyeToy }\end{array}$ & $\begin{array}{l}\text { physical action; } \\
\text { oral } \\
\text { touch } \\
\text { touch; oral } \\
\text { physical action; } \\
\text { oral } \\
\text { physical action } \\
\text { physical action }\end{array}$ & operational \\
\hline enjoying & $\begin{array}{l}\text { sharing pleasure in features such as animation } \\
\text { moving to the music on a CD player }\end{array}$ & $\begin{array}{l}\text { oral; laughter } \\
\text { physical action }\end{array}$ & $\begin{array}{l}\text { learning dispositions } \\
\text { knowledge of the world, } \\
\text { learning dispositions }\end{array}$ \\
\hline explaining & what is on slides for the computer microscope & oral & knowledge of the world \\
\hline instructing & $\begin{array}{l}\text { reading dialogue box on screen } \\
\text { tell child how to use digital camera } \\
\text { tell child to push button on tape player }\end{array}$ & $\begin{array}{l}\text { oral } \\
\text { oral, gesture } \\
\text { oral }\end{array}$ & operational \\
\hline managing & intervening in turn-taking & $\begin{array}{l}\text { oral; facial } \\
\text { expression }\end{array}$ & learning dispositions \\
\hline modelling & $\begin{array}{l}\text { putting on headphones to check sound level } \\
\text { using a play phone to order a taxi }\end{array}$ & $\begin{array}{l}\text { physical action; } \\
\text { oral } \\
\text { physical action; } \\
\text { oral }\end{array}$ & $\begin{array}{l}\text { operational } \\
\text { knowledge of the world }\end{array}$ \\
\hline monitoring & moving child to appropriate level of difficulty & gesture; oral & $\begin{array}{l}\text { learning dispositions; } \\
\text { operational }\end{array}$ \\
\hline prompting & $\begin{array}{l}\text { suggesting a child tries something new } \\
\text { helping with typing in names (typically to start a } \\
\text { new game) }\end{array}$ & $\begin{array}{l}\text { oral } \\
\text { oral; typing }\end{array}$ & $\begin{array}{l}\text { learning dispositions } \\
\text { operational }\end{array}$ \\
\hline $\begin{array}{l}\text { providing } \\
\text { feedback }\end{array}$ & $\begin{array}{l}\text { giving encouragement for efforts } \\
\text { smiling as child types name on keyboard } \\
\text { says 'That's beautiful' when child shows picture on } \\
\text { camera }\end{array}$ & $\begin{array}{l}\text { oral } \\
\text { facial } \\
\text { expression } \\
\text { oral }\end{array}$ & learning dispositions \\
\hline supporting & $\begin{array}{l}\text { stays close to child using video camera for safety } \\
\text { and emotional support }\end{array}$ & $\begin{array}{l}\text { physical } \\
\text { presence }\end{array}$ & $\begin{array}{l}\text { learning dispositions; } \\
\text { operational }\end{array}$ \\
\hline
\end{tabular}


Figure 1 Diagrammatic representation of interaction: Media Interaction Chart

From Plowman (1992, p.275)

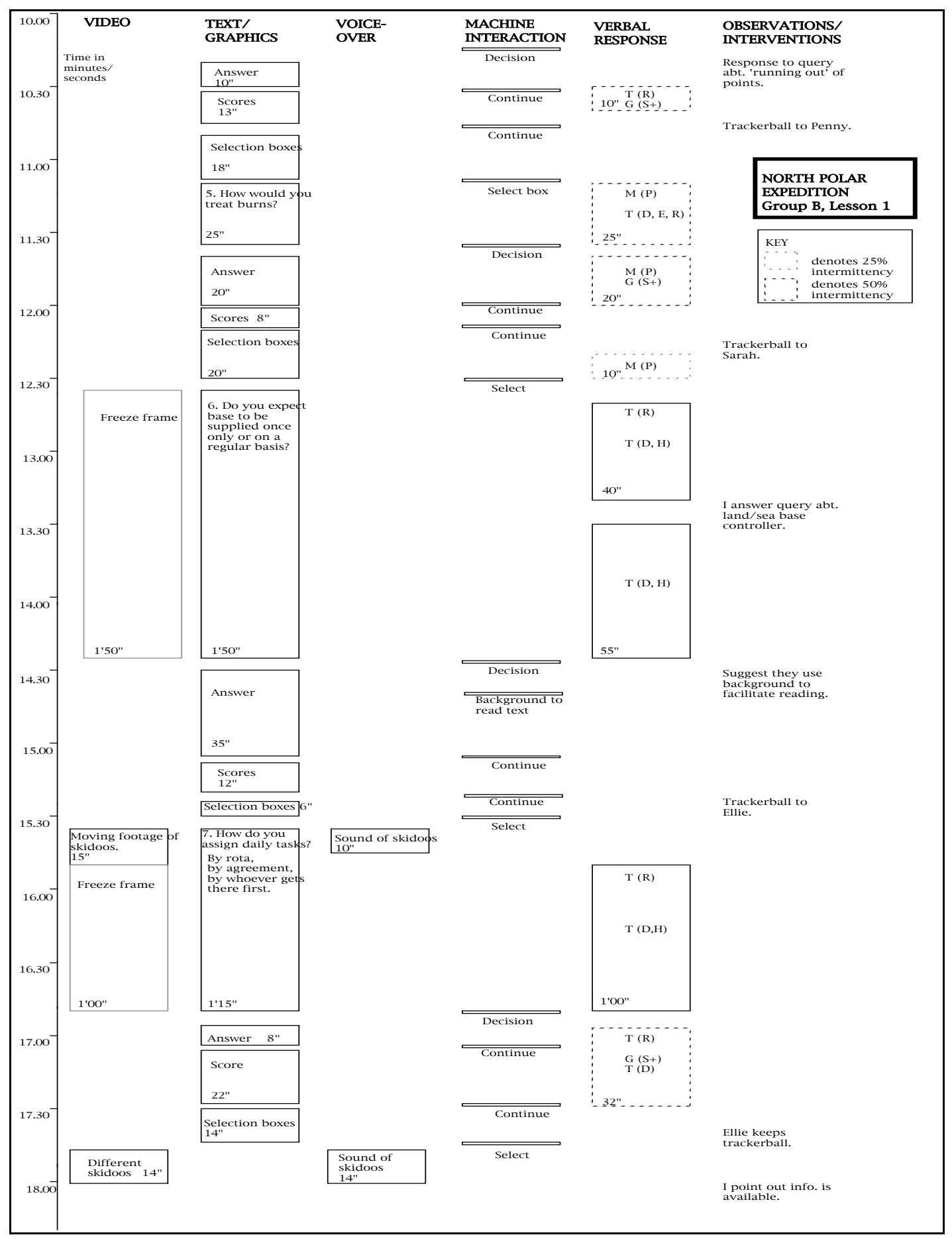


Figure 2 Pictorial representation of interaction: gesture

From Kendon (2004, p. 322)

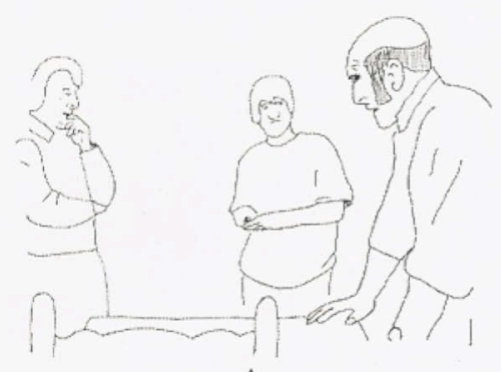

A

In me father's shop,

he used to have sent down from London, a Christmas cake...

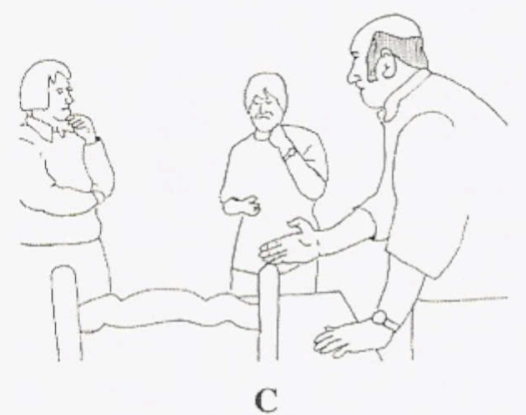

and he'd cut it off in bits

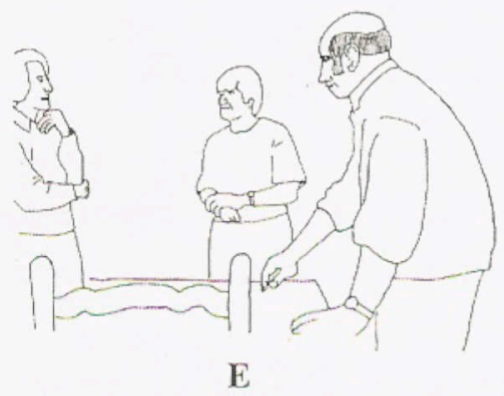

and he'd cut you off a pound and a half

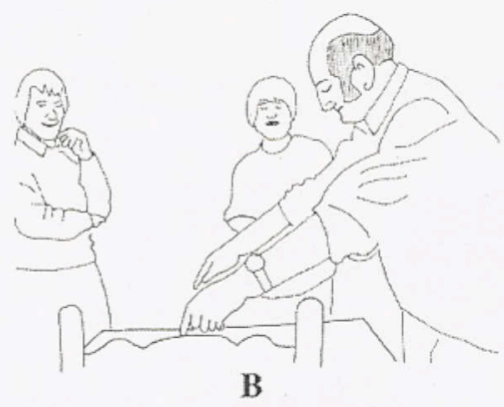

and it was (1.02) this sort of (0.4) size

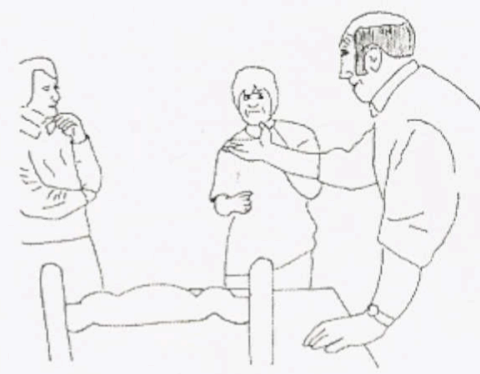

D

and you'd go in and say:

"Can I have a pound and a half?"

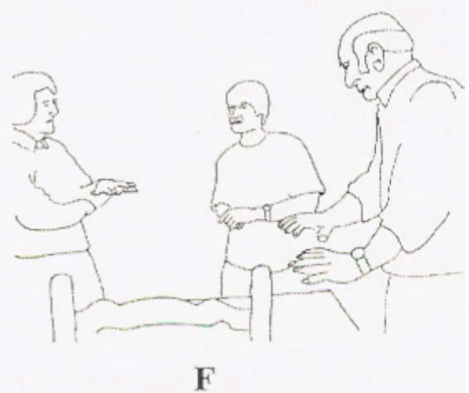

A: And that was ready iced, was it? M: Decorated. the lot!

Fig. 15.6 M explains the Chrstmas cake and how customers could buy pieces of it. For an explanation see text. 
Figure 3 Pictorial representation of interaction: in the classroom From Rogoff (2003, p. 59)

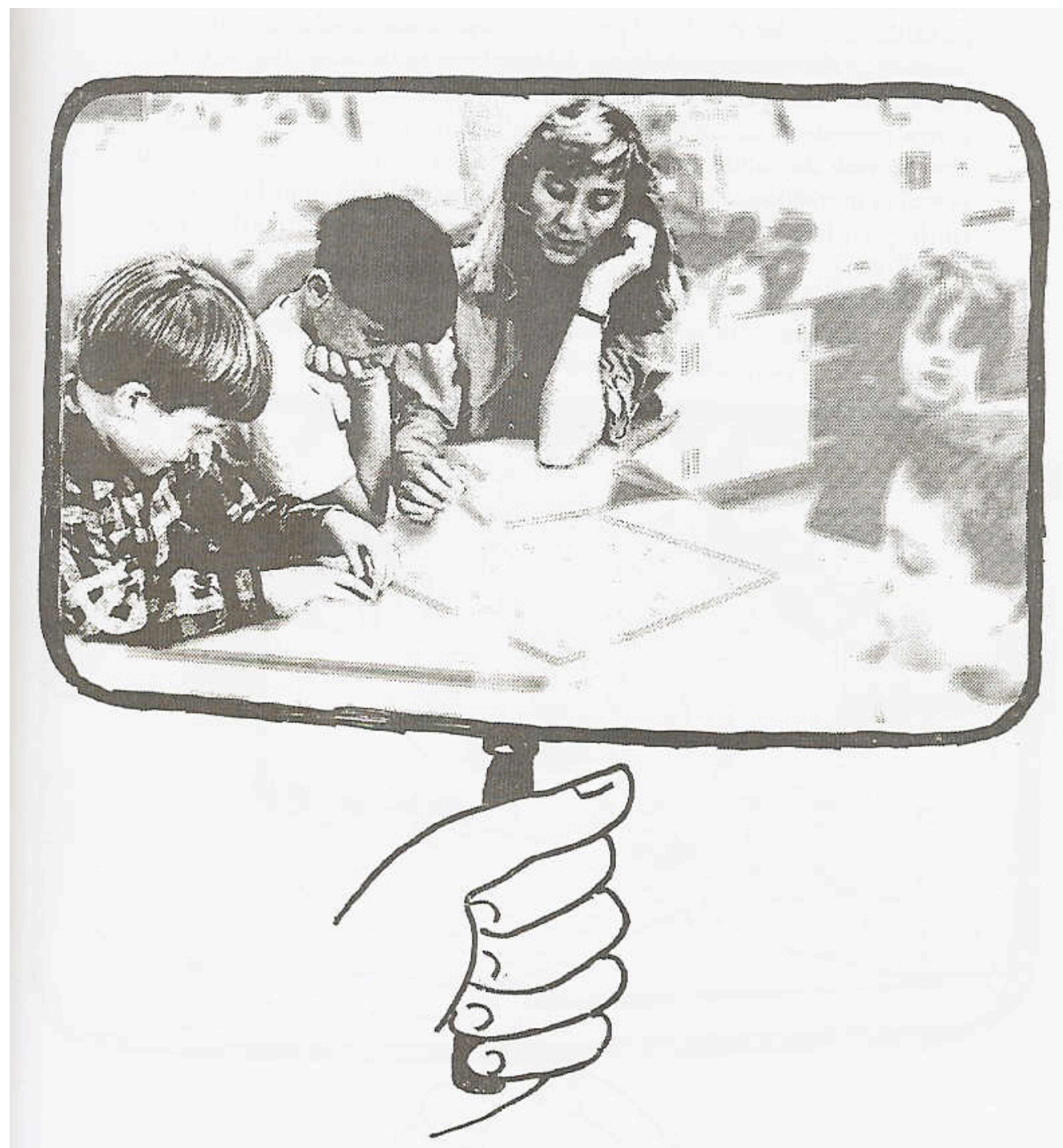




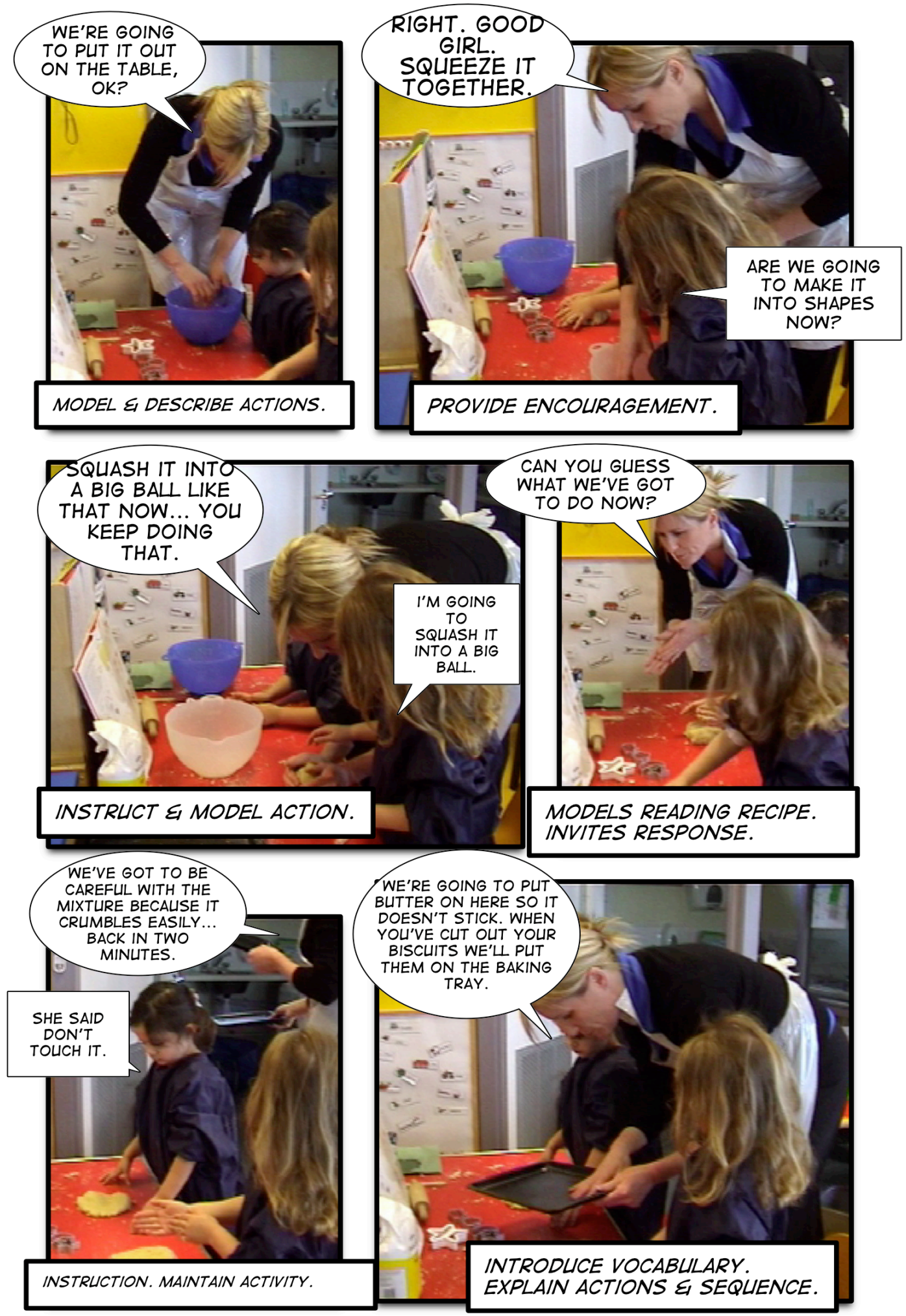

Figure 4

Pictorial representation of interaction: Making cookies 
Figure 5 Pictorial representation of interaction: At the listening centre
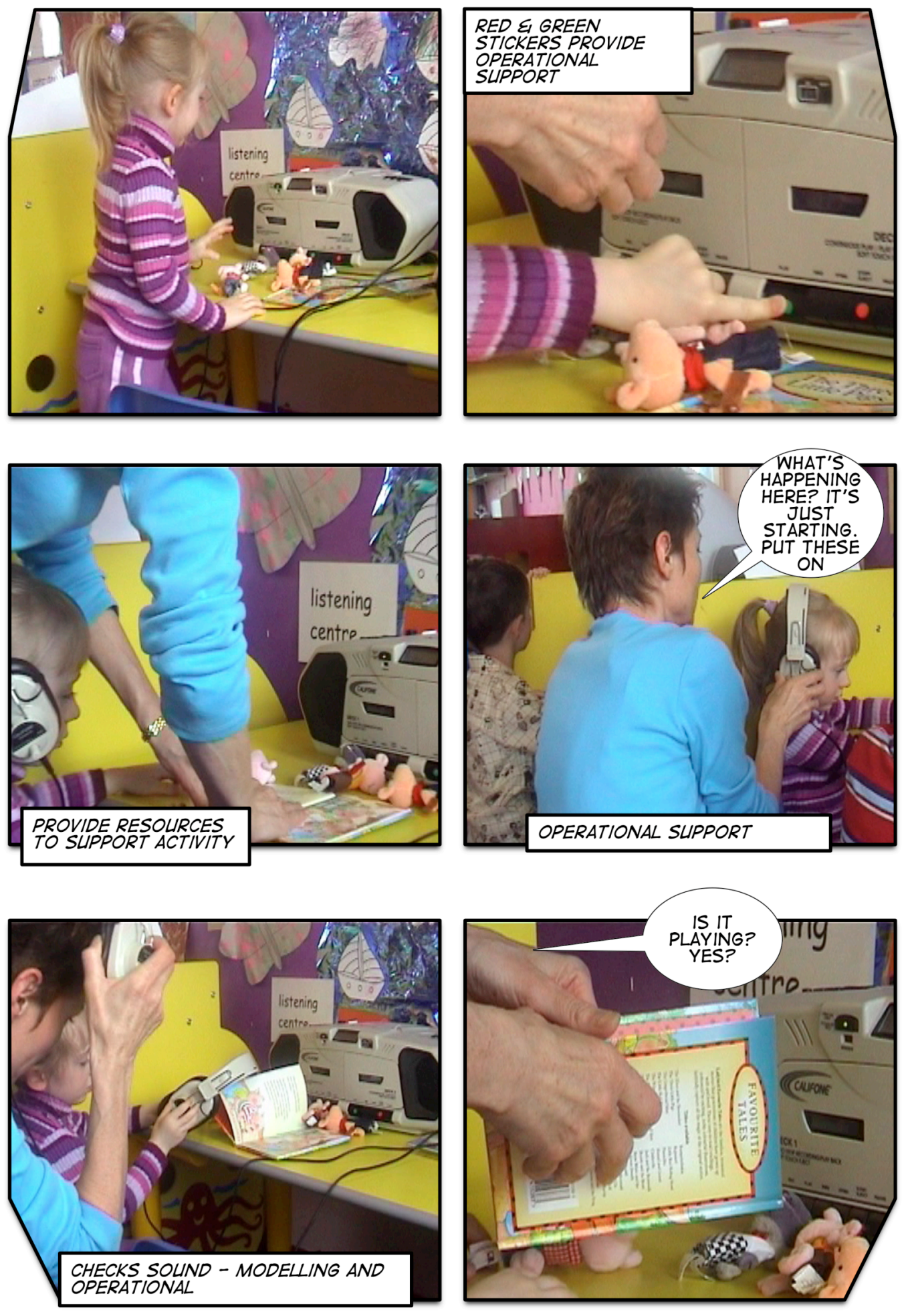